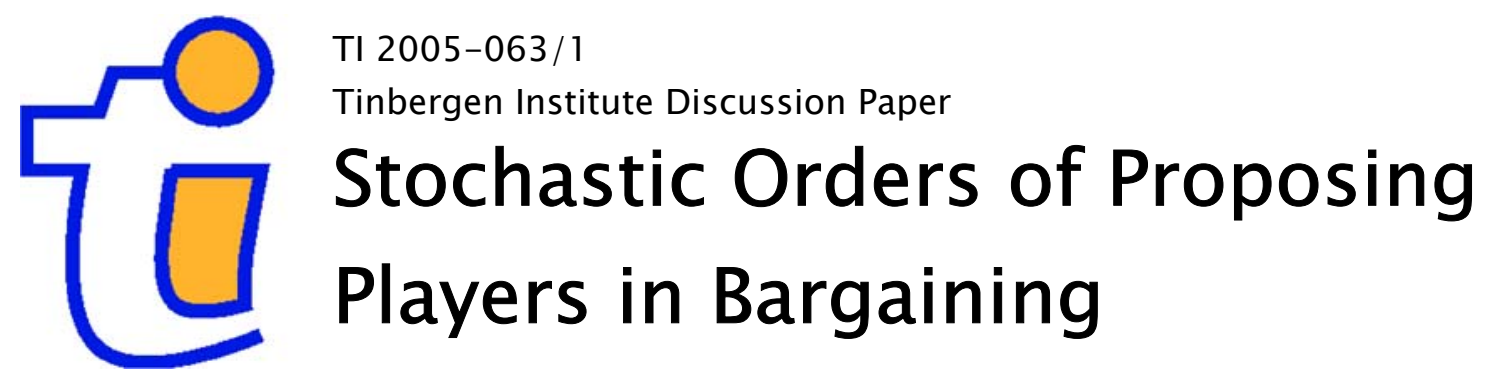

\author{
Harold Houba
}

Faculty of Economics and Business Administration, Vrije Universiteit Amsterdam, and Tinbergen Institute. 


\section{Tinbergen Institute}

The Tinbergen Institute is the institute for economic research of the Erasmus Universiteit Rotterdam, Universiteit van Amsterdam, and Vrije Universiteit Amsterdam.

Tinbergen Institute Amsterdam

Roetersstraat 31

1018 WB Amsterdam

The Netherlands

Tel.: $\quad+31(0) 205513500$

Fax: $\quad+31(0) 205513555$

Tinbergen Institute Rotterdam

Burg. Oudlaan 50

3062 PA Rotterdam

The Netherlands

Tel.: $\quad+31(0) 104088900$

Fax: $\quad+31(0) 104089031$

Please send questions and/or remarks of nonscientific nature to driessen@tinbergen.nl.

Most TI discussion papers can be downloaded at http://www.tinbergen.nl. 


\title{
Stochastic orders of proposing players in bargaining*
}

\author{
Harold Houba ${ }^{\dagger}$ \\ Department of Econometrics and OR \\ and \\ Tinbergen Institute \\ Vrije Universiteit \\ De Boelelaan 1105 \\ $1081 \mathrm{HV}$ Amsterdam \\ The Netherlands
}

June, 2005

\begin{abstract}
The bargaining model with stochastic order of proposing players is properly embedded in continuous time and it is strategically equivalent to the alternating offers model. For all parameter values, the pair of equilibrium proposals corresponds to the Nash bargaining solution of a modified bargaining problem and the Maximum Theorem implies convergence to the Nash bargaining solution when time between proposals vanishes. The model unifies alternating offers, one-sided offers and random proposers. Only continuous-time Markov processes are firmly rooted in probability theory and offer fundamentally different limit results.
\end{abstract}

JEL Classification: C72 Noncooperative Games, C73 Stochastic and Dynamic Games, C78 Bargaining Theory

Keywords: Bargaining, Negotiation, Alternating offers, Markov process, subgame perfect equilibrium, Nash bargaining solution, Maximum Theorem

*The author is indebted to Ad Ridder and Rein Nobel for their advice on continuous-time Markov processes. The usual disclaimer applies.

${ }^{\dagger}$ Department of Econometrics and OR, Vrije Universiteit, De Boelelaan 1105, 1081 HV Amsterdam, Netherlands, Phone: +31 20598 6014, Fax: +31 20598 6020, Email: hhouba@sow.vu.nl, URL: http://staff.feweb.vu.nl/hhouba/. 


\section{Introduction}

Nash (1953) started the research agenda of finding mutually reinforcing axiomatic bargaining solutions and equilibrium outcomes of strategic bargaining procedures. At present, the best known equivalence is between the axiomatic Nash bargaining solution (NBS) and the limit of the unique equilibrium outcome in the strategic alternating offers model with discounting when the time between bargaining rounds vanishes, see e.g. Binmore et al. (1986). The limit bargaining weights depend upon the players' time preferences and every individually rational and Pareto efficient utility pair can be sustained as the limit outcome by an appropriate choice of these preferences. However, the limit outcome and bargaining weights lack any quantification in terms of the bargaining procedure.

Bargaining procedures can be quantified by the stochastic bargaining procedure proposed in Merlo and Wilson (1995), see also Binmore (1987b), Merlo and Wilson (1998) and Muthoo (1999), where integer-like $\{0,1\}$ branches of the game tree in the alternating offers model are replaced by quantifiable state-dependent moves by nature governing the stochastic order of proposing players. Stochastic orders of proposing players include as special cases the alternating offers in Rubinstein (1982), the random proposing players in Hoel (1987) and Muthoo (1999), and the one-sided offers. ${ }^{1}$ Due to the generality and complexity of the model in Merlo and Wilson (1995), who also assume a stochastic surplus, ${ }^{2}$ attention mainly focussed upon stationary SPE outcomes and sufficient conditions for SPE outcomes featuring uniqueness and Pareto efficient delays. It seems that the generality and complexity of this model diverged attention away from the issue of limit results for vanishing time between rounds. Such limit results are at the heart of this paper. However, in understanding some of the well-known bargaining models it turns out that the seemingly innocuous issue of modelling the limit of stochastic orders matters. For that reason, we focus on the standard

\footnotetext{
${ }^{1}$ In their survey, Houba and Bolt (2002) investigate the limit of a less general stochastic process for the ad hoc introduced class of continuous-time Markov processes, but their analysis requires substantial revision because their framework fails to include the bargaining models from the literature and they do not realize that the risk of breakdown might not vanish in the limit. So, they report (22) below instead of (24).

${ }^{2}$ Furusawa and Wen (2002) assume a stochastic surplus modelled as a stochastic disagreement point.
} 
class of bargaining problems in utility representation.

A prerequisite of any analysis is a precise embedding of stochastic orders in continuous time. Insisting on a class of stochastic processes that includes the bargaining procedures mentioned, it suffices to assume transition probabilities that are continuous functions in the time between proposals, especially in the limit when the time between rounds goes to zero. Even in the limit, the mentioned bargaining procedures require positive transition probabilities on the current and other states. However, this violates the underlying definition of transition probabilities as conditional probabilities indicating the likelihood of a transition to another state during a single time interval between two subsequent rounds conditional upon being in the current state. In the limit as this interval shrinks to an 'instant' of time, this conditional probability becomes the probability that the state is in another state at the 'end' of this instant while the state is in a particular state at the 'start' of this instant. Since a state cannot be in a flux between states at each instant of time, limit transition probabilities to another state have to be zero. Only the subclass of continuous-time Markov processes, as surveyed in e.g. Karlin and Taylor (1975), obeys this fundamental property and is properly embedded in probability theory when the time between proposals vanishes. As an independent side matter on transition probabilities, we also argue that the common discounting as assumed in Merlo and Wilson (1995) is automatically captured by a positive risk of inefficient breakdown.

One main result establishes the strategic equivalence between the stochastic order bargaining model and the standard alternating offers model. This immediately establishes a unique subgame perfect equilibrium (SPE) in stationary strategies. Furthermore, the equivalence requires a continuous transformation of the transition probabilities into the standard players' risk of breakdown equal to the probability of becoming the next proposer relative to the probability that the current proposer looses his privilege to propose (to either his opponent or a permanent breakdown). So, we obtain an explicit formula expressing the players' bargaining positions in terms of the transition probabilities of a switch in the pro- 
posers (quantifying the bargaining procedure) and the exogenous risk of breakdown (the 'time preferences'). Another property states that it is advantageous to be the proposing player.

For the characterization of limit SPE outcomes we propose a novel and transparent method that is based upon the equivalence between the fixed point problem for the standard alternating offers model and the Nash bargaining solution of a modified convex bargaining problem in which the Pareto frontier shifts outward and coincides with the proposer's best response functions. This equivalence holds for both zero and positive time between bargaining rounds. Thus, the familiar fixed point satisfies the three axioms for the asymmetric Nash's bargaining solution in Kalai (1977) for this modified bargaining problem. This equivalence is powerful, because it allows to invoke the Maximum Theorem that automatically establishes the existence and convergence to the limit equilibrium outcome given by the asymmetric Nash bargaining solution corresponding to the limit of the modified bargaining problem. For all stochastic processes, except for the subclass of continuous-time Markov processes, the limit bargaining problem is the initial bargaining problem. For the latter subclass, the transformed risks of breakdown in the equivalent alternating offers model remain positive in the limit and depend upon the relative speed by which the underlying transition probabilities out of the current state vanish. For small but positive time between proposals, this subclass of processes has transition probabilities out of the current state that are very small in absolute magnitude, but conditional on a switch out of the current state, the responding player faces a relatively high risk to end up in the worst state. As a consequence, the advantage of being the proposer remains in the limit of continuous-time Markov processes.

This note is organized as follows. In Section 2, the bargaining model with a stochastic order is embedded in continuous time and four special cases are introduced. The equivalence of the stochastic order model and the standard alternating offers model is derived in Section 4, which establishes uniqueness in SPE strategies. The subsequent section deals with the limit results. Since the equivalence between the fixed point problem in the standard alternating 
offers model and the maximization of the Nash product in a modified bargaining problem has some interest of its own, it is presented separately in Section 3.

\section{The bargaining procedure}

Consider two players, indexed $i=1,2$. The subject of their negotiations is the essential and convex bargaining problem in utility representation $(S, d)$, where $S \in \mathbb{R}^{2}$ is a nonempty, compact, strongly comprehensive and convex set, $d \in S$ is the the disagreement point and there exists an $s \in S$ such that $s>d$. Without loss of generality, assume $S=S^{I R}$ where $S^{I R}=\{s \in S \mid s \geq d\}$. Billera and Bixby (1973) show that these bargaining problems are firmly rooted within two-person exchange economies. The Pareto frontier of $S$ can be described by the curve $s_{i}=f_{i}\left(s_{j}\right), i, j=1,2, i \neq j$, where $f_{i}$ is a strictly decreasing and concave function. Furthermore, $f_{2}$ is the inverse function of $f_{1}$ and visa versa.

Time is treated as a continuous variable, but the actual negotiations only take place at equidistant points in time, called bargaining rounds that are indexed $t \in \mathbb{N}$. The time elapsing between two bargaining rounds is denoted as $\Delta \geq 0$, where we explicitly include 0 for the purpose of taking the limit $\Delta$ goes to 0 . A homogeneous Markov process governs which player proposes at round $t$ and whether the negotiations permanently break down. So, there are three states, indexed $k=1,2,3$. State $k=i, i=1,2$, at round $t$ is the transitional state indicating player $i$ is the proposing player at this round who proposes $s^{t} \in S$ and his opponent, denoted $j, j=1,2$ and $j \neq i$, is the responding player who either accepts or rejects player $i$ 's proposal. State $k=3$ is an absorbing state representing perpetual disagreement.

The probability that the stochastic process switches from state $k$ at round $t$ to state $k^{\prime}$ at round $t+1$ is given by the stationary transition probability $p_{k k^{\prime}}(\Delta) \in[0,1]$. The assumptions made impose the following matrix $P(\Delta)$ of transition probabilities:

$$
P(\Delta)=\left[\begin{array}{ccc}
1-p_{12}(\Delta)-p_{13}(\Delta) & p_{12}(\Delta) & p_{13}(\Delta) \\
p_{21}(\Delta) & 1-p_{21}(\Delta)-p_{23}(\Delta) & p_{23}(\Delta) \\
0 & 0 & 1
\end{array}\right]
$$

As mentioned, the properties of these bargaining models at $\Delta=0$ with vanishing risk of 
breakdown are of special interest. Therefore, we assume that the 'limit' matrix at $\Delta=0$ exists and has the following form

$$
P(0)=\left[\begin{array}{ccc}
1-\bar{p}_{12} & \bar{p}_{12} & 0 \\
\bar{p}_{21} & 1-\bar{p}_{21} & 0 \\
0 & 0 & 1
\end{array}\right], \quad \bar{p}_{12}, \bar{p}_{21} \in[0,1] .
$$

We assume the following.

Assumption 1 Each element of $P(\Delta)$ is a continuous function in $\Delta \geq 0, P(\Delta)$ has a structure similar as in (1) and $P(0)$ as in (2).

At later stages of the analysis, we impose the stronger assumption of continuously differentiable functions at $\Delta=0$. In that case, there exists a $3 \times 3$ matrix $Q$ whose $\left(k, k^{\prime}\right)$-element $q_{k k^{\prime}}$ is equal to $p_{k k^{\prime}}^{\prime}(0)$. Obviously, $p_{3 k^{\prime}}^{\prime}(\Delta)=0$ for all $\Delta \geq 0$ and the $Q$-matrix is of the form

$$
Q=\left[\begin{array}{ccc}
q_{11} & q_{12} & q_{13} \\
q_{21} & q_{22} & q_{23} \\
0 & 0 & 0
\end{array}\right]
$$

Assumption 2 Each element of $P(\Delta)$ is a continuously differentiable function in $\Delta \geq 0$, $P(\Delta)$ has a structure similar as in (1), $P(0)$ as in (2), $Q$ as in (3) and $q_{i j}+q_{i 3}>0$, $i, j=1,2, j \neq i$.

Four special cases are of interest, each satisfying Assumption 2: Three bargaining models and a class of continuous-time Markov processes popular in Operations Research. In order to describe these cases, we denote player $i$ 's subjective rate of time preferences as $r_{i} \geq 0$, $i=1,2$, with the convention that we drop the subscript in case of common time preferences. The four cases are:

1. Alternating offers with discounting as in Rubinstein (1982) assumes

$$
P^{A}(\Delta)=\left[\begin{array}{ccc}
0 & e^{-r_{2} \Delta} & 1-e^{-r_{2} \Delta} \\
e^{-r_{1} \Delta} & 0 & 1-e^{-r_{1} \Delta} \\
0 & 0 & 1
\end{array}\right], \quad Q^{A}=\left[\begin{array}{ccc}
0 & -r_{2} & r_{2} \\
-r_{1} & 0 & r_{1} \\
0 & 0 & 0
\end{array}\right]
$$


2. Random proposing players with discounting in Binmore (1987b), ${ }^{3}$ Muthoo (1999) and (the symmetric case) in Hoel (1987) assumes

$$
P^{R}(\Delta)=\left[\begin{array}{ccc}
q_{1} e^{-r_{2} \Delta} & q_{2} e^{-r_{2} \Delta} & 1-e^{-r_{2} \Delta} \\
q_{1} e^{-r_{1} \Delta} & q_{2} e^{-r_{1} \Delta} & 1-e^{-r_{1} \Delta} \\
0 & 0 & 1
\end{array}\right], \quad Q^{R}=\left[\begin{array}{ccc}
-q_{1} r_{2} & -q_{2} r_{2} & r_{2} \\
-q_{1} r_{1} & -q_{2} r_{1} & r_{1} \\
0 & 0 & 0
\end{array}\right],
$$

where $q_{1}+q_{2}=1$ and $q_{1}, q_{2} \geq 0$.

3. One-sided offers (by player 1) with discounting assumes

$$
P^{O}(\Delta)=\left[\begin{array}{ccc}
e^{-r \Delta} & 0 & 1-e^{-r \Delta} \\
e^{-r \Delta} & 0 & 1-e^{-r \Delta} \\
0 & 0 & 1
\end{array}\right], \quad Q^{O}=\left[\begin{array}{ccc}
-r & 0 & r \\
-r & 0 & r \\
0 & 0 & 0
\end{array}\right]
$$

4. Continuous-time Markov processes as in e.g. Karlin and Taylor (1975) assumes

$$
P^{M}(0)=I, \quad Q^{M}, \quad \text { where } q_{k k}^{M} \leq 0 \text { and } q_{k k^{\prime}}^{M} \geq 0, k^{\prime} \neq k
$$

The restrictions on $Q^{M}$ follow from $P^{M}(0)=I$, because this property implies $p_{k k}^{M}(\Delta) \leq$ 1 is nonincreasing for $\Delta>0$ sufficiently close to 0 , while $p_{k k^{\prime}}^{M}(\Delta) \geq 0, k^{\prime} \neq k$, is nondecreasing.

These special cases make clear that the boundary $p_{12}(\Delta)=0, p_{21}(\Delta) \in[0,1]$ for $\Delta \geq 0$ and its reverse case should be part of any analysis, which includes $\bar{p}_{12}=0, \bar{p}_{21} \in[0,1]$ or $\bar{p}_{12} \in[0,1], \bar{p}_{21}=0$. Some terminology from the literature on continuous-time Markov processes also captures these boundaries. A state $k$ is called stable if $p_{k k}(0)=1$ and $q_{k k}$ is finite, whereas, a state $k$ is called instantaneous if $p_{k k}(0)=1$ and $q_{k k}$ infinite. The Markov process is called stable if all states are stable, which can only hold for matrices of the form $P(0)=I$.

A serious matter of concern is that the mathematically well-defined $P(0)$ lacks a fundamental probability-theoretic property underlying continuous-time Markov processes at $\Delta=0$. To see this, the transition probability $P_{i j}(\Delta)$ is implicitly defined as the conditional

\footnotetext{
${ }^{3}$ Binmore (1987b) assumes random proposing players with different probabilities in even and odd bargaining rounds, which fall outside the class of stochastic processes introduced in Section 2 except when probabilities in odd and even rounds coincide.
} 
probability $\operatorname{Pr}(k(\Delta)=j \mid k(0)=i)$, where $k(\Delta)$ and $k(0)$ denote the state at time 0 and $\Delta$. As $\Delta$ goes to 0 , the conditional probability converges to $\operatorname{Pr}(k(0)=j \mid k(0)=i)$. The limit transition probabilities of the continuous-time Markov processes have the property that $P(0)=I$, see e.g. Karlin and Taylor (1975). This makes perfect sense: At each instant of time, the stochastic process is in exactly one state. So, conditional on being in state $k=i$, the conditional probability distribution over all states at the same instant contracts all probability mass on the current state. Therefore, any $P(0) \neq I$ describes a limit stochastic process that is in some kind of flux between several states like in quantum mechanics. Then, at any moment in time, it is not clear what role each player has, what the status of each proposal is and what acceptance of a proposal means. Continuous-time Markov processes assume that it takes some positive (stochastic) time to leave a state. Nevertheless, we proceed with stochastic processes featuring $P(0) \neq I$ in order to unify the convergence results in the literature, but we see these results as purely mathematical lacking a probability theoretic foundation.

Bargaining models are typically analyzed under the assumption of a 'shrinking cake' property for positive time between proposals, which holds in case of positive risks of breakdown. Furthermore, we assume vanishing risk of breakdown as $\Delta$ goes to 0 .

Assumption $3 p_{13}(\Delta), p_{23}(\Delta)>0$ for all $\Delta>0$ and $p_{13}(0)=p_{23}(0)=0$.

This assumption slightly differs from the setup in Merlo and Wilson (1995) and Merlo and Wilson (1998), who additionally assume some common discount factor $\beta<1$, which is regarded as essential in order to have a shrinking cake property or contraction. However, Assumption 3 and an essential bargaining problem suffice to obtain a contraction, which would also be present in case either $p_{13}(\Delta)$ or $p_{23}(\Delta)$ is zero (but not both). Furthermore, discounting by $\beta$ is equivalent to the following compound lottery: First, Nature draws with probability $\beta$ the outcome 'continue' and with $1-\beta$ the outcome permanent breakdown with payoff vector 0 as if it were a second disagreement point. If the realization is 'continue', then Nature draws the new state by the stochastic process $P(\Delta)$. Either we set $\beta=1$ and neglect 
this parameter (which avoids specifying the function $\beta(\Delta)$ for $\Delta \geq 0$ ), or we extend $P(\Delta$ ) by introducing a second absorbing state and reduce the compound lottery to the simple lottery over four states. ${ }^{4}$ Note that the simple lotteries (4)-(6) can also be reformulated as state-dependent compound lotteries with 'discount factor' $\beta_{k}(\Delta)=p_{k 3}(\Delta)>0$, for $\Delta>0$, and second disagreement point equal to $d$. State-dependent simple lotteries over states are all that matter.

\section{Alternating offers as a single convex program}

In this section, we establish the equivalence between the set of fixed points for the standard alternating offers model and the maximizer of a single convex program in which the objective is the asymmetric Nash product. In deriving limit results in Section 5 we will rely on the properties of this program, but the equivalence also has merits on its own. Throughout this section we assume that $P(\Delta)$ is given by (4) and we write $\delta_{i}, i=1,2$, for $e^{-r_{i} \Delta} \in(0,1]$ whenever convenient.

The main results derived in this section are illustrated by means of the following wellknown example. Consider the divide-the-dollar with transferable utility and common time preferences. Formally, $d=0, f_{2}\left(s_{1}\right)=1-s_{1}$ for $s_{1} \in[0,1], r_{1}=r_{2}=r$ meaning $\delta_{1}=$ $\delta_{2}=\delta$ and $\delta=1$ corresponding to $\Delta=0$. The SSPE utility pairs $x=\left(x_{1}, 1-x_{1}\right)$ and $y=\left(1-y_{2}, y_{2}\right)$ solve the fixed point problem

$$
\left.\begin{array}{l}
x_{1}=1-\delta y_{2}, \\
y_{2}=1-\delta x_{1},
\end{array}\right\} \quad \Rightarrow \quad x_{1}=y_{2}=\frac{1}{1+\delta}
$$

The solution Figure 1 provides a graphical illustration in the $\left(s_{1}, s_{2}\right)$-space of the curves $s_{1}=1-\delta s_{2}$ and $s_{2}=1-\delta s_{1}$ and the Nash product $\left(s_{1}-d_{1}\right)^{\frac{1}{2}}\left(s_{2}-d_{2}\right)^{\frac{1}{2}} \operatorname{through}\left(\frac{1}{1+\delta}, \frac{1}{1+\delta}\right)$. Obviously, $\left(x_{1}, y_{2}\right)$ maximizes this Nash product under the restrictions $s_{1} \leq 1-\delta s_{2}$ and $s_{2} \leq 1-\delta s_{1}$. Moreover, it coincides with the intersection of these curves. Since this maximization program is strictly convex for all $\delta \in[0,1]$, the Maximum Theorem implies

\footnotetext{
${ }^{4}$ This compound lottery is also present in the general model in Merlo and Wilson (1995) and is equivalent to a reduced simple lottery with positive state-dependent mass on an absorbing inefficient state.
} 


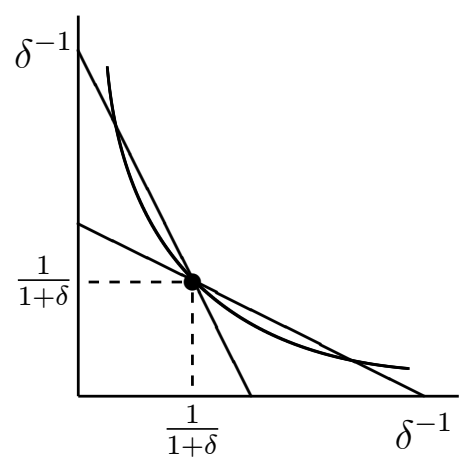

Figure 1: $\left(x_{1}, y_{2}\right)$ in (8) maximizes the Nash product $\left(s_{1}-d_{1}\right)^{\frac{1}{2}}\left(s_{2}-d_{2}\right)^{\frac{1}{2}}$.

that the maximizer is unique and is continuous in the parameter $\delta$ (which includes $\Delta=0$ ). The main result in this section generalizes this insight.

Before deriving the main result of this section, we briefly summarize some relevant results for the standard alternating offers model. For $\Delta>0$, there exists a unique SPE in stationary strategies. Such strategies feature a pair of state-dependent continuation SSPE payoffs $x, y \in$ $S$, where $x(y)$ refers to state $k=1(k=2)$. In the SSPE, player 1's SSPE proposal $s^{1}(y)$ solves

$$
s^{1}(y) \in \arg \max _{s \in S} s_{1}, \quad \text { s.t. } \quad s_{1} \leq f_{1}\left(s_{2}\right), s_{2} \geq\left(1-\delta_{2}\right) d_{2}+\delta_{2} y_{2} .
$$

In the unique optimum (which exists), player 1 strictly prefers to make an acceptable offer, both constraints are binding (which implies Pareto efficiency), and, by definition of the continuation payoff, $x=s^{1}(y)$. Similar,

$$
y=s^{2}(x)=\arg \max _{s \in S} s_{2}, \quad \text { s.t. } \quad s_{2} \leq f_{2}\left(s_{1}\right), s_{1} \geq\left(1-\delta_{1}\right) d_{1}+\delta_{1} x_{1} .
$$

The binding constraints imply the familiar fixed point problem given by

$$
\begin{array}{ll}
x_{1}=f_{1}\left(y_{2}\right), & x_{2}=\left(1-\delta_{2}\right) d_{2}+\delta_{2} y_{2}, \\
y_{1}=\left(1-\delta_{1}\right) d_{1}+\delta_{1} x_{1}, & y_{2}=f_{2}\left(y_{1}\right) .
\end{array}
$$

Furthermore, in every fixed point $(x, y)$ of (11) the SSPE continuation payoffs $x$ and $y$ have the same (asymmetric) Nash product given by

$$
\left(x_{1}-d_{1}\right)^{\alpha}\left(x_{2}-d_{2}\right)^{1-\alpha}=\delta_{1}^{\alpha}\left(x_{1}-d_{1}\right)^{\alpha}\left(y_{2}-d_{2}\right)^{1-\alpha}=\left(y_{1}-d_{1}\right)^{\alpha}\left(y_{2}-d_{2}\right)^{1-\alpha},
$$


where $\alpha \equiv \alpha\left(\delta_{1}, \delta_{2}\right)=\ln \delta_{2}\left(\ln \delta_{1}+\ln \delta_{2}\right)^{-1}=r_{2}\left(r_{1}+r_{2}\right)^{-1} \in[0,1]$ for all $\Delta \in[0, \infty)$, provided $\left(r_{1}, r_{2}\right) \neq(0,0)$, see e.g. Binmore et al. (1986), Osborne and Rubinstein (1990), van Damme (1991), Muthoo (1999) and Houba and Bolt (2002). Therefore, we assume $\left(r_{1}, r_{2}\right) \neq(0,0)$ in this section whenever bargaining weights enter the analysis, except in Lemma 4 and 5 below. Houba (1993) exploits (12) to establish a general proof for (11) admitting a unique fixed point.

In contrast to the equalities in fixed point problem (11), we focus on the four constraints in (9) and (10) that enter as the constraints in the single convex program. Elementary substitutions that preserve the inequality signs (because $f_{i}, i=1,2$, decreasing) half the number of variables and constraints:

$$
\begin{aligned}
& x_{1} \leq f_{1}\left(\left(1-\delta_{2}\right) d_{2}+\delta_{2} y_{2}\right), \\
& y_{2} \leq f_{2}\left(\left(1-\delta_{1}\right) d_{1}+\delta_{1} x_{1}\right)
\end{aligned}
$$

These two inequality constraints can be represented in the $\left(s_{1}, s_{2}\right)$-space. Each constraint describes the proposing player's best response given his opponent's threshold level. Similar as in Nash's demand game, these best-response functions coincide with the Pareto frontier of $S$ at the 'non-perturbed' situation $\delta_{1}=\delta_{2}=1$ and each shifts above $S$ for the perturbation $\left(\delta_{1}, \delta_{2}\right) \neq(1,1)$ allowing for a unique 'Nash equilibrium', see e.g. Binmore (1987a) and, especially, van Damme (1991). Under additional individual rationality, (13) defines the set of feasible utility pairs

$$
S\left(\delta_{1}, \delta_{2}\right)=\left\{s \geq d \mid s_{1} \leq f_{1}\left(\left(1-\delta_{2}\right) d_{2}+\delta_{2} s_{2}\right), s_{2} \leq f_{2}\left(\left(1-\delta_{1}\right) d_{1}+\delta_{1} s_{1}\right)\right\}
$$

The constraints are also well-defined for either $\delta_{1}$ or $\delta_{2}$ (or both) equal to 1 (where 'both' coincides with $\Delta=0$ ). For our purposes, the following result is relevant.

Lemma 4 For all $\left(\delta_{1}, \delta_{2}\right) \in(0,1]^{2}, S\left(\delta_{1}, \delta_{2}\right)$ is a nonempty, compact and convex set. Moreover, $S \subseteq S\left(\delta_{1}, \delta_{2}\right)$ and $s \leq\left(f_{1}\left(d_{2}\right), f_{2}\left(d_{1}\right)\right)$ for all $s \in S\left(\delta_{1}, \delta_{2}\right)$.

Proof.

First, $f_{1}$ and $f_{2}$ decreasing imply $f_{1}\left(s_{2}\right) \leq f_{1}\left(\left(1-\delta_{2}\right) d_{2}+\delta_{2} s_{2}\right) \leq f_{1}\left(d_{2}\right)$ and $f_{2}\left(s_{1}\right) \leq$ 
$f_{2}\left(\left(1-\delta_{1}\right) d_{1}+\delta_{1} s_{1}\right) \leq f_{2}\left(d_{1}\right)$, which proves the 'moreover' part and $S\left(\delta_{1}, \delta_{2}\right)$ is nonempty and bounded. The weak inequalities imply $S\left(\delta_{1}, \delta_{2}\right)$ is closed. Finally, $f_{1}$ and $f_{2}$ concave imply convexity.

Since every fixed point of (11) corresponds one-to-one to the intersection points of the two curves describing the Pareto frontier of $S\left(\delta_{1}, \delta_{2}\right)$ in $(14)$, we define the set of intersection points as

$$
I\left(\delta_{1}, \delta_{2}\right)=\left\{s \geq d \mid s_{1}=f_{1}\left(\left(1-\delta_{2}\right) d_{2}+\delta_{2} s_{2}\right), s_{2}=f_{2}\left(\left(1-\delta_{1}\right) d_{1}+\delta_{1} s_{1}\right)\right\} .
$$

Obviously, $I\left(\delta_{1}, \delta_{2}\right)$ consists of a single intersection point that is contained in $S\left(\delta_{1}, \delta_{2}\right)$, which is stated in the following lemma. ${ }^{5}$

Lemma 5 For all $\left(\delta_{1}, \delta_{2}\right) \in(0,1]^{2}, I\left(\delta_{1}, \delta_{2}\right) \subseteq S\left(\delta_{1}, \delta_{2}\right)$ consists of a unique element.

Figure 1 suggests the equivalence between a unique intersection point and the unique maximizer of the Nash product $(9)$ over $S\left(\delta_{1}, \delta_{2}\right)$. However, uniqueness of the maximizer has not been established. Therefore, we define the set of maximizers as

$$
M\left(\delta_{1}, \delta_{2}\right)=\arg \max _{s}\left(s_{1}-d_{1}\right)^{\alpha}\left(s_{2}-d_{2}\right)^{1-\alpha}, \quad \text { s.t. } \quad s \in S\left(\delta_{1}, \delta_{2}\right),
$$

where $\alpha=\alpha\left(\delta_{1}, \delta_{2}\right) \in[0,1]$. By Lemma 4, this program is convex and well-defined and straightforward application of the Maximum Theorem, see e.g. Varian (1992), implies the following result.

Lemma 6 Let $\left(r_{1}, r_{2}\right) \neq(0,0)$. Then, $M\left(\delta_{1}, \delta_{2}\right)$ is a nonempty, compact, convex-valued and upper semi-continuous correspondence in $\delta_{1}$ and $\delta_{2}\left(\right.$ or $r_{1}, r_{2}$ and $\Delta$ ).

The main step consists of relating the set of maximizers to the set of intersection points and, simultaneously, showing that the set of maximizers consists of a unique element for $\Delta>0$. The following theorem establishes that the insight of Figure 1 generally holds.

\footnotetext{
${ }^{5} \mathrm{~A}$ proof without reference to the properties of fixed point problem (11) would be based upon application of the Mean Value Theorem for the rectangle given by given by $s_{1} \geq d_{1}, s_{1} \leq \delta_{1}^{-1} f_{1}\left(d_{2}\right)-\delta_{1}^{-1}\left(1-\delta_{1}\right) d_{1}$, $s_{2} \geq d_{2}$ and $s_{2} \leq \delta_{2}^{-1} f_{2}\left(d_{1}\right)-\delta_{2}^{-1}\left(1-\delta_{2}\right) d_{2}$.
} 
Theorem 7 Let $\left(r_{1}, r_{2}\right) \neq(0,0)$. Then, $M\left(\delta_{1}, \delta_{2}\right) \subseteq I\left(\delta_{1}, \delta_{2}\right)$. If additionally $\Delta>0$, then $M\left(\delta_{1}, \delta_{2}\right)=I\left(\delta_{1}, \delta_{2}\right)$ consists of a single element.

Proof.

First, consider $\Delta=0$. Then $\delta_{1}=\delta_{2}=1$ implies that $I(1,1)$ coincides with the Pareto frontier of $S$. For every $\alpha(1,1) \in[0,1]$, the optimum of program (16) coincides with the Nash bargaining solution, which is unique even for $\alpha(1,1)=0$ or $\alpha(1,1)=1$. Hence, the singleton $M(1,1)$ is a strict subset of $I(1,1)$.

Second, consider $\Delta>0, r_{1}=0$ and $r_{2}>0$. Then $\delta_{1}=1$ and $\delta_{2}<1$ and $\alpha\left(1, \delta_{2}\right)=1$. So, $S\left(1, \delta_{2}\right)=S$ and $I\left(1, \delta_{2}\right)=M\left(1, \delta_{2}\right)=\left\{\left(f_{1}\left(d_{2}\right), d_{2}\right)\right\}$. This also establishes the case $r_{1}>0$ and $r_{2}=0$.

Finally, consider $r_{1}, r_{2}, \Delta>0$. Then $\left(\delta_{1}, \delta_{2}\right) \in(0,1)^{2}$ and, thus, $\alpha\left(\delta_{1}, \delta_{2}\right) \in(0,1)$. In essence, we reformulate the proof in e.g. Houba (1993). According to this proof: For an arbitrary fixed point of (11) represented in $S$, the strictly quasi-concave Nash product curve and the Pareto frontier of $S$ intersect twice at the distinct points $x$ and $y$ (note $\left.y_{1}=\left(1-\delta_{1}\right) d_{1}+\delta_{1} x_{1}<x_{1}\right)$, namely where the 'high end' enters $S$ at $y$ and where the 'low end' leaves $S$ at $x$. Thus, the tangent of the Nash product curve at $y=\left(y_{1}, f_{2}\left(y_{1}\right)\right)$ is steeper than any tangent of the Pareto frontier described by $f_{2}$. Similar, the tangent to the Nash product curve at $x=\left(f_{1}\left(x_{2}\right), x_{2}\right)$ is less steep than the tangent of $f_{1}$. These facts imply $s_{2}>f_{2}\left(\left(1-\delta_{1}\right) d_{1}+\delta_{1} s_{1}\right)$ for the 'high end' of the Nash product curve through $\left(x_{1}, y_{2}\right) \in S\left(\delta_{1}, \delta_{2}\right)$, which holds for all $s_{2}>y_{2}$ or, equivalently, all $s_{1}<x_{1}$. By symmetry, $s_{1}>f_{1}\left(\left(1-\delta_{2}\right) d_{2}+\delta_{2} s_{2}\right)$ for all $s_{1}>x_{1}$ on the 'low end' of the Nash product curve through $\left(x_{1}, y_{2}\right) \in S\left(\delta_{1}, \delta_{2}\right)$. So, both 'ends' do not belong to $S\left(\delta_{1}, \delta_{2}\right)$ and the strictly quasi-concave Nash product curve at any arbitrary $\left(x_{1}, y_{2}\right) \in I\left(\delta_{1}, \delta_{2}\right)$ is tangent to $S\left(\delta_{1}, \delta_{2}\right)$. Hence, $\left(x_{1}, y_{2}\right) \in M\left(\delta_{1}, \delta_{2}\right)$ and $I\left(\delta_{1}, \delta_{2}\right) \subseteq M\left(\delta_{1}, \delta_{2}\right)$. Finally, $\alpha\left(\delta_{1}, \delta_{2}\right) \in(0,1)$ implies program (16) is strictly convex and admits a unique maximizer. Lemma 9 implies $I\left(\delta_{1}, \delta_{2}\right)=M\left(\delta_{1}, \delta_{2}\right)$ are both singletons. 
From the proof it follows that particular parameter values induce either $\alpha=0$ or $\alpha=$ 1 and these values destroy the strict convexity of program (16), but never its convexity. Nevertheless, the maximizer is always unique. Theorem 7 implies the following strengthening of Lemma 6.

Theorem 8 Let $\left(r_{1}, r_{2}\right) \neq(0,0)$. The unique $\left(x_{1}\left(\delta_{1}, \delta_{2}\right), y_{2}\left(\delta_{1}, \delta_{2}\right)\right) \in M\left(\delta_{1}, \delta_{2}\right)$ is continuous in $\delta_{1}$ and $\delta_{2}\left(\right.$ or $r_{1}, r_{2}$ and $\left.\Delta\right)$. Moreover, $\left(x_{1}(1,1), y_{2}(1,1)\right)$ exists and coincides with the Nash bargaining solution with bargaining weight $\alpha=r_{2}\left(r_{1}+r_{2}\right)^{-1}$.

A similar continuity result also holds for the parameter $d \in S$. Furthermore, the advantage of being the proposing player vanishes in the limit, because $\left(x_{1}(1,1), f_{2}\left(x_{1}(1,1)\right)\right)=$ $\left(f_{1}\left(y_{2}(1,1)\right), y_{2}(1,1)\right)$.

The continuity also ensures that the limit solution exists in case either $\Delta$ or one (but not both) of the $r_{i}$ 's goes to infinity. Furthermore, this last theorem is more general than existing convergence results, because it also allows for arbitrary continuous functions $\delta_{i}(\Delta)$ provided $\ln \delta_{2}(\Delta)\left(\ln \delta_{1}(\Delta)+\ln \delta_{2}(\Delta)\right)^{-1}$ exists for all $\Delta \in[0, \infty)$, which is the approach taken in Section 5.

The optimum of program (16) yields the Nash bargaining solution for the modified bargaining problem with $S\left(\delta_{1}, \delta_{2}\right)$ as the modified feasible set. Therefore, the three axioms of Pareto efficiency, Independence of Irrelevant Alternatives and Invariance of Affine Transformations in Kalai (1977) immediately axiomatizes the class of optima of program (16) for all parameter values, not just at $\Delta=0$.

\section{Uniqueness in SPE strategies}

In this section, we establish for the stochastic order model the uniqueness in subgame perfect equilibrium (SPE) strategies for fixed and positive time between negotiation rounds. The key argument shows that the stochastic order bargaining is strategically equivalent to standard alternating offers and, therefore, the player-dependent proposals can be written as the convex 
program (16). Since time between rounds is positive and fixed throughout this section we drop $\Delta>0$ from our notation.

The starting point of the analysis is the derivation of a SSPE with the state-dependent continuation payoffs $x$ and $y \in S$. At state $k=1$, player 2's expected continuation payoff from rejecting $s \in S$ depends upon the three possible realizations of the stochastic process. Accepting an arbitrary proposal $s \in S$ is a best response for player 2 if

$$
s_{2} \geq p_{13} d_{2}+p_{12} y_{2}+\left(1-p_{12}-p_{13}\right) x_{2}
$$

and player 1's best possible agreement is given by

$$
s^{1}(x, y) \in \arg \max _{s \in S} s_{1}, \quad \text { s.t. } \quad s_{1} \leq f_{1}\left(s_{2}\right), p_{13} d_{2}+p_{12} y_{2}+\left(1-p_{12}-p_{13}\right) x_{2} \leq s_{2} .
$$

As in Section 3, player 1 strictly prefers to propose the unique optimum of $(18), x=s^{2}(x, y)$ and both constraints are binding. Maintaining the inequalities in the optimum, we write

$$
x_{1} \leq f_{1}\left(x_{2}\right), \quad x_{2} \geq p_{13} d_{2}+p_{12} y_{2}+\left(1-p_{12}-p_{13}\right) x_{2}
$$

These inequalities can be further reduced, but first we define $\delta_{2} \equiv \delta_{2}\left(p_{12}, p_{13}\right)=\frac{p_{12}}{p_{12}+p_{13}} \in$ $[0,1)$, which is a continuous function in $p_{12} \in[0,1]$ and $p_{13}>0$. Then solving the linear constraint for $x_{2}$ and, next, substitution of the solution into the nonlinear constraint (which preserves the inequality signs) yields

$$
x_{1} \leq f_{1}\left(\left(1-\delta_{2}\right) d_{2}+\delta_{2} y_{2}\right)
$$

Similar, for the continuous function $\delta_{1} \equiv \delta_{1}\left(p_{21}, p_{23}\right)=\frac{p_{21}}{p_{21}+p_{23}} \in[0,1)$ we obtain

$$
y_{2} \leq f_{2}\left(\left(1-\delta_{1}\right) d_{1}+\delta_{1} x_{1}\right)
$$

Obviously, the last two (in)equalities form fixed point problem (11) that admits a unique fixed point $\left(x_{1}, y_{2}\right)$ for all $\left(\delta_{1}, \delta_{2}\right) \in(0,1]^{2},\left(\delta_{1}, \delta_{2}\right) \neq(1,1)$. Since some bargaining models impose $p_{i j}=0$ and, therefore, $\delta_{j}=0$, we must extend the analysis a little further. Without loss of generality, $\delta_{1} \in[0,1)$ and $\delta_{2}=0$ imply that the unique fixed point of (11) 
is $\left(f_{1}\left(d_{2}\right), f_{2}\left(\left(1-\delta_{1}\right) d_{1}+\delta_{1} f_{1}\left(d_{2}\right)\right)\right)$. So, there exists a unique fixed point $\left(x_{1}, y_{2}\right)$ for all $\left(\delta_{1}, \delta_{2}\right) \in[0,1]^{2},\left(\delta_{1}, \delta_{2}\right) \neq(1,1)$.

Uniqueness in SPE strategies is established by applying the method in Shaked and Sutton (1984). Formally, denote $M^{1} \in S$ as player 1's best SPE outcome in state $k=1$ and $m^{2}$ as 2's worst SPE outcome in state $k=2$. Then, player 1's utility $M_{1}^{1}$ is maximal when $M^{1}$ solves the following program

$$
M^{1} \in \arg \max _{s^{t} \in S} s_{1}^{t}, \quad \text { s.t. } \quad f_{1}\left(s_{2}^{t}\right) \geq s_{1}^{t}, s_{2}^{t} \geq p_{13} d_{2}+p_{12} m_{2}^{2}+\left(1-p_{12}-p_{13}\right) M_{2}^{1},
$$

and, by similar arguments as before, we obtain

$$
M_{1}^{1} \leq f_{1}\left(\left(1-\delta_{2}\right) d_{2}+\delta_{2} m_{2}^{2}\right)
$$

By symmetry and minimizing player 2's utility $m_{2}^{2}$ in order to obtain player 2's worst SPE outcome we solve

$$
m^{2} \in \arg \min _{s^{t} \in S} s_{2}^{t}, \quad \text { s.t. } \quad s_{2}^{t} \geq f_{2}\left(s_{1}^{t}\right), s_{1}^{t} \leq p_{23} d_{1}+p_{21} M_{1}^{1}+\left(1-p_{21}-p_{23}\right) m_{1}^{2},
$$

which yields

$$
m_{2}^{2} \geq f_{2}\left(\left(1-\delta_{1}\right) d_{1}+\delta_{1} M_{1}^{1}\right)
$$

Again, the familiar arguments for the standard alternating offers model apply, see e.g. Osborne and Rubinstein (1990), van Damme (1991), Muthoo (1999) and Houba and Bolt (2002), and we obtain $M^{1}=m^{2}=\left(x_{1}, y_{2}\right)$. By symmetry, similar results hold for the other two bounds.

The results thus far imply that the stochastic order bargaining model is strategically equivalent to the standard alternating offers model and only requires a proper transformation from the probabilities in (1) into the risks of breakdown in the alternating offers model. The following result summarizes these findings.

Theorem 9 Let $\left(\delta_{1}\left(p_{21}, p_{23}\right), \delta_{2}\left(p_{12}, p_{13}\right)\right) \in[0,1]^{2} \backslash\{(1,1)\}$. The unique SPE strategies in the stochastic order bargaining model are stationary, player 1's proposal in state $k=1$ is 
$x=\left(x_{1}, f_{2}\left(x_{1}\right)\right)$, player 2's proposal in state $k=2$ is $y=\left(f_{1}\left(y_{2}\right), y_{2}\right)$ and each proposal is immediately accepted. Moreover, the pair $\left(x_{1}, y_{2}\right)$ is the unique pair that solves (20) and (21) with equal signs.

This theorem provides the following intuition. In state $i=1,2$, the responding player $j$ 's perceived probability of a next round is equal to $\delta_{j}\left(p_{i j}, p_{i 3}\right) \in[0,1)$. This probability is the conditional probability of reaching player $j$ 's most preferred state $k=j$ with probability $p_{i j}$ conditional on leaving the less favorable state $k=i$ with probability $p_{i j}+p_{i 3}$. Obviously, $\delta_{j}\left(p_{i j}, p_{i 3}\right)$ is increasing in the probability $p_{i j}$ of reaching the most preferred state $k=j$ and decreasing in the probability $p_{i 3}$ of ending up in his worst state $k=3$. In that respect, one-sided offers with $i$ as the proposing player $\left(p_{i j}=0\right)$ is the worst possible bargaining situation for the responding player $j$, which includes the take-it-or-leave-it or ultimatum bargaining situation with $p_{i 3}=1$ as a special case. Note also that an increase in $p_{i j}$ shortens the expected wait for player $j$ to become the proposing player. These findings confirm that it is advantageous to have the initiative to propose in negotiations.

For the special cases we obtain the following:

1. Alternating offers features $p_{i j}^{A}+p_{i 3}^{A}=1$ and, therefore, $\delta_{j}^{A}=e^{-r_{j} \Delta}>0, j=1,2$. The bargaining weight $\alpha\left(\delta_{1}^{A}, \delta_{2}^{A}\right)=r_{2}\left(r_{1}+r_{2}\right)^{-1}$, which is well-defined except at $r_{1}=r_{2}=$ 0 .

2. Random proposing players. Then $\delta_{1}^{R}=\frac{\delta_{1} q_{1}}{1-\delta_{1}\left(1-q_{1}\right)}=\frac{\delta_{1} q_{1}}{1-\delta_{1} q_{2}}$ and $\delta_{2}^{R}=\frac{\delta_{2} q_{2}}{1-\delta_{2}\left(1-q_{2}\right)}=\frac{\delta_{2} q_{2}}{1-\delta_{2} q_{1}}$, where $\delta_{j}=e^{-r_{j} \Delta}, j=1,2$. Moreover, $\alpha\left(\delta_{1}^{R}, \delta_{2}^{R}\right)$ is well-defined, because either $\delta_{1}^{R}$ or $\delta_{2}^{R}$ is positive. In particular, $q_{1}=q_{2}=\frac{1}{2}$ and $r_{1}=r_{2}=r$ imply $\delta_{1}^{R}=\delta_{2}^{R}=\frac{\delta}{2-\delta}$, where $\delta=e^{-r \Delta}$.

3. One-sided offers (by player 1) implies $\delta_{1}^{O}=e^{-r \Delta}>0$ and $\delta_{2}^{O}=0$. Player 1 has all the bargaining power because $\lim _{\delta_{2} \rightarrow 0} \alpha\left(\delta_{1}^{O}, \delta_{2}\right)=1$.

4. Continuous-time Markov processes imply $\delta_{1}^{M}=p_{12}^{M}\left(p_{12}^{M}+p_{13}^{M}\right)^{-1}<1$ and $\delta_{2}^{M}=$ 
$p_{21}^{M}\left(p_{21}^{M}+p_{23}^{M}\right)^{-1}<1$. The weight $\alpha\left(\delta_{1}^{M}, \delta_{2}^{M}\right)$ is well-defined, provided $\left(p_{12}^{M}, p_{21}^{M}\right) \neq$ $(0,0)$.

In Section 5, limit results are derived based upon properties of the single convex program (16) as stated in Theorem 8. Since some bargaining models impose $p_{i j}=0$ and, therefore, $\delta_{j}=0$, we need to extend the equivalence to this convex program and this theorem. As mentioned, for $\delta_{1} \in(0,1)$ and $\delta_{2}=0$ the unique fixed point of (11) is $\left(f_{1}\left(d_{2}\right), f_{2}\left(\left(1-\delta_{1}\right) d_{1}+\delta_{1} f_{1}\left(d_{2}\right)\right)\right)$. However, $\lim _{\delta_{2} \rightarrow 0} \alpha\left(\delta_{1}, \delta_{2}\right)=1$ for all $\delta_{1} \in(0,1)$, in $(16)$ yields the convex set of maximizers given by $s_{1}=f_{1}\left(d_{2}\right)$ and $s_{2} \in\left[d_{2}, f_{2}\left(\left(1-\delta_{1}\right) d_{1}+\delta_{1} f_{1}\left(d_{2}\right)\right)\right]$, which is a nondegenerate interval. Although the equivalence with program (16) breaks down at the border cases, we mention that the unique fixed point of (11) corresponds to any limit point as (positive) $\delta_{2}$ goes to 0 . The following result extends Theorem 8 and is a direct consequence of the Maximum Theorem for convex programs, see e.g. Varian (1992). This result still suites our purposes in Section 5.

Theorem 10 Let $\left(\delta_{1}, \delta_{2}\right) \in[0,1]^{2},\left(\delta_{1}, \delta_{2}\right) \neq(0,0),(1,1)$. Program (16) is a convex program and its set of maximizers $\left\{\left(x_{1}\left(\delta_{1}, \delta_{2}\right), y_{2}\left(\delta_{1}, \delta_{2}\right)\right)\right\}$ is a nonempty, compact, convexvalued and upper semi-continuous correspondence in $\left(\delta_{1}, \delta_{2}\right)$. Moreover, for $\left(\delta_{1}, \delta_{2}\right) \in(0,1]^{2}$, $\left(\delta_{1}, \delta_{2}\right) \neq(1,1)$, the correspondence is a continuous function and, by upper semi-continuity,

$$
\begin{aligned}
\lim _{\delta_{1} \rightarrow 0}\left(x_{1}\left(\delta_{1}, \delta_{2}\right), y_{2}\left(\delta_{1}, \delta_{2}\right)\right) & =\left(f_{1}\left(\left(1-\delta_{2}\right) d_{2}+\delta_{2} f_{2}\left(d_{1}\right)\right), f_{2}\left(d_{1}\right)\right) \\
\lim _{\delta_{2} \rightarrow 0}\left(x_{1}\left(\delta_{1}, \delta_{2}\right), y_{2}\left(\delta_{1}, \delta_{2}\right)\right) & =\left(f_{1}\left(d_{2}\right), f_{2}\left(\left(1-\delta_{1}\right) d_{1}+\delta_{1} f_{1}\left(d_{2}\right)\right)\right)
\end{aligned}
$$

Continuity at $(0,0)$ or $(1,1)$ is only ensured for sequences of $\left(\delta_{1}, \delta_{2}\right)$ such that $\lim _{\left(\delta_{1}, \delta_{2}\right) \rightarrow(0,0)} \alpha\left(\delta_{1}, \delta_{2}\right)$, respectively, $\lim _{\left(\delta_{1}, \delta_{2}\right) \rightarrow(1,1)} \alpha\left(\delta_{1}, \delta_{2}\right)$ exist, which is one of the main issues in deriving limit results in Section 5. Since $\delta_{1}=\delta_{1}\left(p_{12}, p_{13}\right)$ and $\delta_{2}=\delta_{2}\left(p_{21}, p_{23}\right)$ are continuous in the transition probabilities, we immediately obtain upper semi-continuity in transition probabilities and, under the additional restriction $p_{12}, p_{21}>0$, continuity. This implies that the equilibrium outcomes gradually change in these probabilities, especially, if one moves for 
instance from alternating offers to one-sided offers implying that there is no abrupt change or sensitivity to 'small' changes in the game tree.

Finally, as established in Section 3, the optimum of program (16) yields the Nash bargaining solution for the modified bargaining problem with $S\left(\delta_{1}, \delta_{2}\right)$ as the modified feasible set for all parameter values, not just at $\Delta=0$. This interpretation/axiomatization holds for all parameter values whether expressed as $\left(\delta_{1}, \delta_{2}\right)$ or indirectly as the elements of the matrix $P(\Delta)$ with transition probabilities.

\section{$5 \quad$ Limit results}

A well-known result for the standard alternating offer model states the convergence of the SSPE proposals to the Nash bargaining solution as the time between proposals vanishes, see e.g. Binmore et al. (1986). In this section, we characterize such limit outcomes in case of stochastic orders by employing Theorem 10.

Since program (16) is a convex program, we may treat the optimum of this program as a correspondence of $\Delta \geq 0$ whose limit is investigated by letting $\Delta$ go to 0 . The dependence upon $\Delta$ is rather indirect through the transition probabilities in $P(\Delta)$ that specify the reformulated probabilities $\delta_{j}, j=1,2$, in Section 4 and these in turn specify the bargaining weight $\alpha$ of Section 3. To reduce notation, we write $\delta_{j}(\Delta), j=1,2$, for $\delta_{j}\left(p_{i j}(\Delta), p_{i 3}(\Delta)\right)$, $\alpha(\Delta)$ for player 1's bargaining weight $\ln \delta_{2}(\Delta) /\left(\ln \delta_{1}(\Delta)+\ln \delta_{2}(\Delta)\right)$ and $\left(x_{1}(\Delta), y_{2}(\Delta)\right)$ for the fixed point $\left(x_{1}\left(\delta_{1}(\Delta), \delta_{2}(\Delta)\right), y_{2}\left(\delta_{1}(\Delta), \delta_{2}(\Delta)\right)\right)$.

Theorem 10 enhances characterizing the limit solution. First, it implies that it is sufficient to establish continuity of $\delta_{j}$ and $\alpha$ at $\Delta=0$, because then the limit exists and convergence is immediate. At $\Delta=0$, the limit solution corresponds to the optimum of program (16) for $\delta_{1}(0), \delta_{2}(0)$ and $\alpha(0)$ (although some attention should be give to $\delta_{1}(0)=0$ or $\delta_{2}(0)=0$ as discussed in Section 4). In order to structure the discussion, we first investigate the relevant properties of the function $\delta_{j}$, before dealing with $\alpha$. Furthermore, our results are derived under Assumption 2, but our derivation also investigates whether Assumption 1 would be 
sufficient for these properties.

Continuity of $\delta_{j}(\Delta)$ at $\Delta=0$ : Assumption 1 ensures the continuity of the function $\delta_{j}(\Delta)=$ $p_{i j}(\Delta)\left(p_{i j}(\Delta)+p_{i 3}(\Delta)\right)^{-1} \in[0,1)$ on $(0, \infty)$, because $p_{i j}(\Delta) \in[0,1]$ and $p_{i 3}(\Delta)>0$. If $p_{i j}(0)=\bar{p}_{i j}>0$, then continuity also holds at $\Delta=0$ and simple substitution of limit values yields $\delta_{j}(0)=1$. The boundary case $\bar{p}_{i j}=0$ requires the stronger Assumption 2 and application of L'Hôpital's rule to obtain $\delta_{j}(0)=q_{i j}\left(q_{i j}+q_{i 3}\right)^{-1} \in[0,1]$. Therefore, $\delta_{j}:[0, \infty) \rightarrow[0,1]$ is continuous requires Assumption 1 and, additionally, either $\bar{p}_{i j}>0$ or Assumption 2.

Continuity of $\alpha(\Delta)$ at $\Delta=0$ : Continuity of $\alpha(\Delta)$ on $[0, \infty)$ requires at least continuity of the functions $\delta_{j}(\Delta), j=1,2$, but this is not sufficient because our earlier results indicate that $\delta_{1}(0)=\delta_{2}(0)=1$ for the generic class of parameter values and that $\delta_{1}(0)=\delta_{2}(0)=0$ for particular continuous-time Markov processes. Continuity of $\left(\delta_{1}(\Delta), \delta_{2}(\Delta)\right)$ involves three distinct cases: $\left(\bar{p}_{12}, \bar{p}_{21}\right) \in(0,1]^{2}$; the boundary $\bar{p}_{12}=0, \bar{p}_{21} \in(0,1]$ (or its reverse case) and $\left(\bar{p}_{12}, \bar{p}_{21}\right)=(0,0)$.

1. $\left(\bar{p}_{12}, \bar{p}_{21}\right) \in(0,1]^{2}$ implies $\delta_{1}(0)=\delta_{2}(0)=1$ under Assumption 1. So, $\alpha(0)=\frac{0}{0}$. Imposing Assumption 2 and application of L'Hôpital's rule suffices to obtain ${ }^{6}$

$$
\begin{aligned}
\lim _{\Delta \rightarrow 0} \alpha(\Delta) & =\frac{\delta_{2}^{\prime}(0) \delta_{2}^{-1}(0)}{\delta_{1}^{\prime}(0) \delta_{1}^{-1}(0)+\delta_{2}^{\prime}(0) \delta_{2}^{-1}(0)} \\
& =\frac{-q_{13} \bar{p}_{12}^{-1}}{-q_{23} \bar{p}_{21}^{-1}-q_{13} \bar{p}_{12}^{-1}} \\
& =\frac{q_{13} \bar{p}_{21}}{q_{23} \bar{p}_{12}+q_{13} \bar{p}_{21}} \in[0,1]
\end{aligned}
$$

provided $\left(q_{13}, q_{23}\right) \neq(0,0)$. Since $\delta_{j}(0)=1$, only the relative derivatives of $\delta_{j}$ at $\Delta=0$ matter in the limit weight $\alpha(0)$. The bargaining weight in the last line are also obtained in the standard alternating offer model with a risk of breakdown $\delta_{j}=e^{-\Delta q_{i 3} \bar{p}_{j i}}$.

\footnotetext{
${ }^{6}$ This follows from
}

$$
\delta_{j}^{\prime}(0)=\frac{p_{i j}^{\prime}(0) p_{i 3}(0)-p_{i j}(0) p_{i 3}^{\prime}(0)}{\left(p_{i j}(0)+p_{i 3}(0)\right)^{2}}=-\frac{q_{i 3}}{\bar{p}_{i j}}
$$


So, player $i$ 's bargaining position improves if either his limit probability of becoming the proposer increases or if his opponent faces a higher risk of breakdown because $p_{i 3}(\Delta) \approx q_{i 3} \Delta+o(\Delta)$ for sufficiently small $\Delta>0$. Finally, taking either the limit $\bar{p}_{12}$ or $\bar{p}_{21}$ goes to 0 (but not both) is also well-defined and yields $\alpha(0)=1$, respectively, $\alpha(0)=0$.

2. $\bar{p}_{12}=0, \bar{p}_{21} \in(0,1]$ implies $\delta_{1}(0)=1$ and, by Assumption $2, \delta_{2}(0)=q_{12}\left(q_{12}+q_{13}\right)^{-1} \in$ $[0,1]$. The interesting case is $q_{13}>0$ and $q_{12}>0$ with $\delta_{2}(0) \in(0,1) .{ }^{7}$ Then direct substitution yields

$$
\alpha(0)=\frac{\ln \delta_{2}(0)}{\ln \delta_{1}(0)+\ln \delta_{2}(0)}=1,
$$

which implies continuity of $\alpha$ in the parameter $\bar{p}_{12}$ at $\bar{p}_{12}=0$. Furthermore, the limit $q_{12}$ goes to 0 exists and yields $\alpha(0)=1$ at $q_{12}=0$, provided $q_{13}>0$. Also, $q_{12}>0$ and $q_{13}=0$ imply $\alpha(0)=1$.

3. $\left(\bar{p}_{12}, \bar{p}_{21}\right)=(0,0)$. By Assumption 2 we have $\delta_{j}(0)=q_{i j}\left(q_{i j}+q_{i 3}\right)^{-1} \in[0,1], j=1,2$. The novel case that differs from the previous two cases corresponds to $\left(q_{13}, q_{23}\right)>(0,0)$ and, therefore, $\left(\delta_{1}(0), \delta_{2}(0)\right) \in[0,1)^{2}$. If additionally $q_{12}, q_{21}>0$, then $\left(\delta_{1}(0), \delta_{2}(0)\right) \in$ $(0,1)^{2}$ and direct substitution yields

$$
\alpha(0)=\frac{\ln \delta_{1}(0)}{\ln \delta_{1}(0)+\ln \delta_{2}(0)} \in(0,1) .
$$

Letting $q_{12}$ or $q_{21}$ go to 0 (but not both simultaneously) yields $\alpha(0)=1$ and $\alpha(0)=0$, respectively, provided $q_{13}>0$ or $q_{23}>0$.

From these three cases, we conclude that Assumption 2 and L'Hôpital's rule were always invoked. Obviously, Assumption 2 is sufficient, but not necessary. We have arrived at the following convergence result.

Theorem 11 If Assumptions 2 and 3 hold, then $\lim _{\Delta \rightarrow 0}\left\{\left(x_{1}(\Delta), y_{2}(\Delta)\right)\right\}$ of program (16) exists and converges to a subset of maximizers $\left\{\left(x_{1}(0), y_{2}(0)\right)\right\}$ of program $(16)$ for $\left(\delta_{1}(0), \delta_{2}(0)\right)$ $\in[0,1]^{2}$ and $\alpha(0) \in[0,1]$ corresponding to either (22), (23) and (24).

\footnotetext{
${ }^{7}$ The case $q_{13}=0$ implies $\delta_{2}(0)=1$ and is therefore already captured by the previous case.
} 


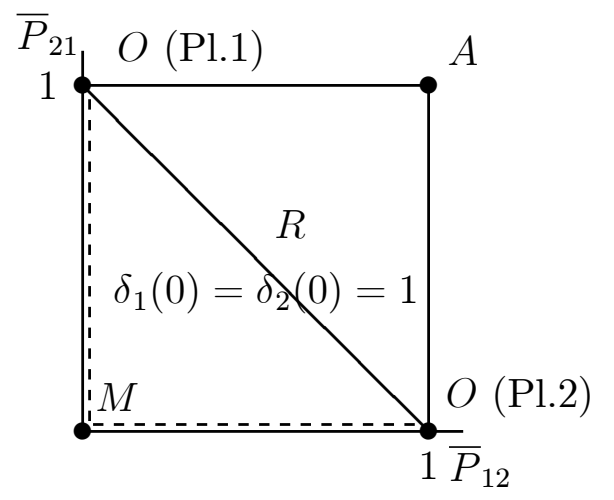

Figure 2: Representation of bargaining models and area $\delta_{1}(0)=\delta_{2}(0)=1$ in $\left(\bar{p}_{12}, \bar{p}_{21}\right) \in$ $[0,1]^{2}$, where $A, R, O$ and $M$ refer to the four special cases.

Figure 2 and 3 illustrate the limit results in the $\left(\bar{p}_{12}, \bar{p}_{21}\right)$ and $\left(\delta_{1}(0), \delta_{2}(0)\right)$ spaces. The first figure indicates the position of the four special cases considered thus far and the area that maps into $\delta_{1}(0)=\delta_{2}(0)=1$, which is the area Northeast of the dashed lines. The dashed border cases and the continuous-time Markov process in the origin required separate analysis in the second and third case treated in front of Theorem 11. The second figure shows the space of discount factors at $\Delta=0$ and how the $\left(\bar{p}_{12}, \bar{p}_{21}\right)$ space is mapped into it. The area Southwest of the dashed lines, indicated by $M$, corresponds to the limit area that can be attained by the continuous-time Markov process. Both figures indicate that the results obtained for continuous-time Markov processes are fundamentally different from those for other processes.

Theorem 11 establishes the convergence to the limit set of outcomes of program (16), but this program specifies convergence to the Nash bargaining solution of the modified bargaining problem with the set of feasible utility pairs equal to $S\left(\delta_{1}(0), \delta_{2}(0)\right)$. The obvious question is: Under what conditions does the Nash bargaining solution for this limit set coincide with the Nash bargaining solution for $(S, d)$ ? Imposing $S\left(\delta_{1}, \delta_{2}\right)=S$ implies that at least one of the $\delta_{j}$ 's should be equal to 1 and, by Figure 3, this means that it suffices to have at least one positive $\bar{p}_{i j}$. So, we only fail convergence to the NBS of $(S, d)$ for the 'island' of continuoustime Markov processes in Figure 2. These results also have consequences with respect to the 


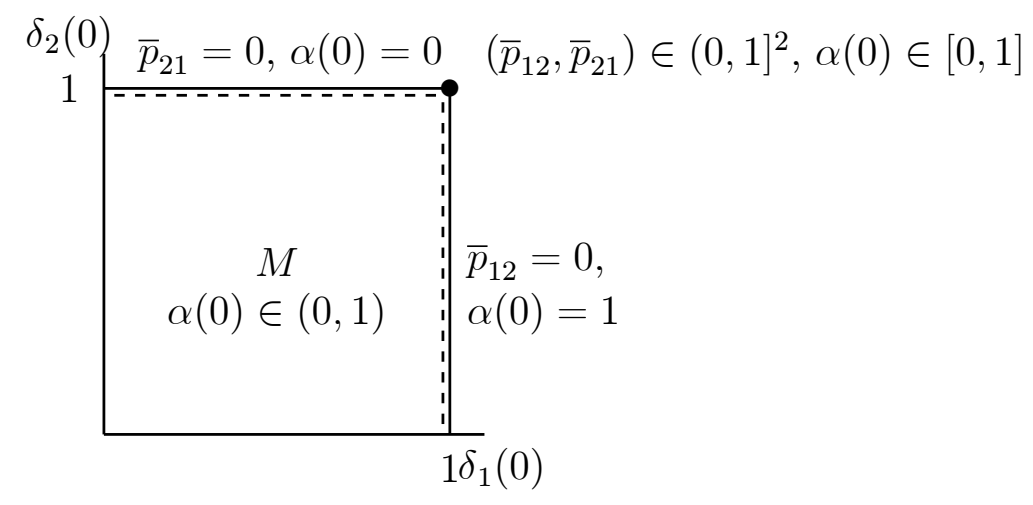

Figure 3: Representation of limit results in $\left(\delta_{1}(0), \delta_{2}(0)\right) \in[0,1]^{2}$, where $M$ refers to the special case $\bar{p}_{12}=\bar{p}_{21}=0$.

advantage of being the proposer in the limit. To see this, recall that the state-dependent equilibrium proposals are $x(0)=\left(x_{1}(0), f_{2}\left(x_{1}(0)\right)\right)$ and $y(0)=\left(f_{1}\left(y_{2}(0)\right), y_{2}(0)\right)$ and, by fixed point problem $(11)$ and $\delta_{2}(\Delta)<1$, we can express the advantage as

$$
x_{1}(\Delta)=f_{1}\left(\left(1-\delta_{2}(\Delta)\right) d_{2}+\delta_{2}(\Delta) y_{2}(\Delta)\right)>f_{1}\left(y_{2}(\Delta)\right)=y_{1}(\Delta), \quad \Delta>0 .
$$

Then $\delta_{2}(0)=1$ implies that the advantage disappears in the limit. However, for all stable continuous-time Markov processes with $q_{13}>0$ we have $\delta_{2}(0)<1$ and, therefore, the state dependent proposals $x(\Delta)$ and $y(\Delta)$ fail a common limit point, i.e., $x(0) \neq y(0)$, meaning the advantage remains present in the limit. Note that either $q_{13}=q_{23}=0$ or, otherwise, either $k=1$ or $k=2$ being an instantaneous state ( $q_{12}$ or $q_{21}$ goes to infinity) is needed to have one of the $\delta_{j}$ 's equal to 1 at $\Delta=0$.

The reason that $\delta_{j}(0)<1$ for stable continuous-time Markov processes is directly related to its property $P(0)=I$. For small $\varepsilon>0$ time between proposals, continuity implies $P(\varepsilon) \approx I$ and $p_{k k^{\prime}}(\varepsilon)=q_{k k^{\prime}} \varepsilon+o(\varepsilon)$. The explanation is not that this process shows some inertia for a switch in states to occur that is reflected in the high expected number of bargaining rounds before a switch occurs. Rather, the conditional probabilities of the 'new' states conditional on a switch in states depends upon the risk of breakdown $p_{i 3}(\varepsilon) \approx q_{i j} \varepsilon$ and $p_{i j}(\varepsilon) \approx q_{i j} \varepsilon$. Therefore, $\delta_{j}(\varepsilon) \approx q_{i j}\left(q_{i j}+q_{i 3}\right)^{-1}$ and, although $p_{i 3}(\varepsilon)$ and $p_{i j}(\varepsilon)$ are very small in absolute magnitude, a switch of states involves a relatively large risk for the 
responding player of ending up in his 'worst' state. These considerations are not relevant for instantaneous states with $q_{i j}=\infty$ or stochastic processes with $\bar{p}_{i j}>0$, because then the jump to the responding player's 'best' state is infinitely many times more likely than the jump to his worst state.

Two remarks are in place. First, these results show that the qualitative results obtained in the alternating and random offers models are robust with respect to alternative bargaining procedures captured by a stochastic process determining the order of proposers. Second, multiplicity of maximizers is restricted to either $\delta_{1}(0) \in(0,1)$ and $\delta_{2}(0)=0$ (or the reverse case), which requires the nongeneric condition $\bar{p}_{12}=\bar{p}_{12}=0$ and either $q_{12}=0$ or $q_{21}=0$. These cases correspond to the axes in Figure 3. So, in the generic case, program (16) yields a unique maximizer.

Finally, for the four special cases mentioned earlier we obtain the following:

1. Alternating offers. Then $\bar{p}_{12}=\bar{p}_{21}=1$ implies $\delta_{1}(0)=\delta_{2}(0)=1$. Then $\delta_{1}^{\prime}(0)=r_{j}$, $j=1,2$, in (17) yields $\alpha(0)=\frac{r_{1}}{r_{1}+r_{2}}$. Moreover, $x(0)=y(0)$.

2. Random proposing players. Consider $q_{1} \in(0,1)$ first. Then $\bar{p}_{12}=\bar{p}_{22}=q_{2}$ and $\bar{p}_{11}=\bar{p}_{21}=q_{1}$ are both positive, which implies $\delta_{1}^{R}(0)=\delta_{2}^{R}(0)=1$. Next, substitution of the parameter values into (22) yields $\alpha(0)=\frac{q_{1} r_{2}}{q_{1} r_{2}+r_{1} q_{2}} \in(0,1)$ as in Binmore (1987b) and Muthoo (1999). In particular, symmetry $q_{1}=q_{2}$ and $r_{1}=r_{2}$ yields the symmetric weight $\alpha(0)=\frac{1}{2}$ as in Hoel (1987). Finally, the border case $q_{1}=0$ is also well defined and results in the extreme bargaining weight $\alpha(0)=0$, which is in line with (23).

3. One-sided offers (by player 1 ). $\delta_{1}(0)=1$ and $\delta_{2}(0)=0$ imply $\alpha(0)=1$. Moreover, $x(0)=y(0)=\left(f_{1}\left(d_{2}\right), d_{2}\right)$.

4. Continuous-time Markov processes. For this special case $\delta_{j}(0)=q_{i j}\left(q_{i j}+q_{i 3}\right)^{-1} \in$ $[0,1], j=1,2$, do not rely on the absolute values of $p_{i j}(0)=0$ and $p_{i 3}(0)=0$, but rather on the slopes by which $p_{i j}(\Delta)$ and $p_{i 3}(\Delta)$ approach $\Delta=0$. As mentioned, the advantage of being a proposers remains in the limit and, thus, $x_{1}(0)>f_{1}\left(y_{2}(0)\right)$. 


\section{Concluding remarks}

The analysis in the previous sections revealed a fundamental problem. Several well-known bargaining models nicely fit the unifying stochastic order bargaining procedure when time between proposals is positive. However, in letting this time go to zero, the interpretation of the limit model is ambiguous and describes a constant flux over several states. Only the 'island' of continuous-time Markov processes, being firmly rooted in probability theory, offers a safe haven but it cannot capture the well-known bargaining models and it yields fundamentally different limit results. These subtle points must be taken care of in any future analysis of limit outcomes in the general model proposed in Merlo and Wilson (1995).

\section{References}

Billera, L. and R. Bixby (1973). A characterization of Pareto surfaces. Proceedings American Mathematical Society 41, 261-267.

Binmore, K. (1987a). Nash bargaining theory II. In K. Binmore and P. Dasgupta (Eds.), The economics of bargaining, pp. 61-76. Oxford: Basil Blackwell.

Binmore, K. (1987b). Perfect equilibria in bargaining models. In K. Binmore and P. Dasgupta (Eds.), The economics of bargaining, pp. 77-105. Oxford: Basil Blackwell.

Binmore, K., A. Rubinstein, and A. Wolinsky (1986). The Nash bargaining solution in economic modeling. Rand Journal of Economics 17, 176-188.

Furusawa, T. and Q. Wen (2002). Bargaining with stochastic disagreement payoffs. International Journal of Game Theory 31, 571-591.

Hoel, M. (1987). Bargaining games with a random sequence of who makes the offers. Economics Letters 24, 5-9.

Houba, H. (1993). An alternative proof of uniqueness in noncooperative bargaining. Economics Letters 41, 253-256. 
Houba, H. and W. Bolt (2002). Credible threats in negotiations; A game theoretic approach. Theory and Decision Library. Series C: Game Theory Mathematical Programming and Operations Research, vol. 32. Norwell, Mass. and Dordrecht: Kluwer Academic.

Kalai, E. (1977). Nonsymmetric Nash solutions and replication of 2-person bargaining. International Journal of Game Theory 6, 129-133.

Karlin, S. and H. Taylor (1975). A first course in stochastic processes. New York: Academic press.

Merlo, A. and C. Wilson (1995). A stochastic model of sequential bargaining with complete information. Econometrica 63, 371-399.

Merlo, A. and C. Wilson (1998). Efficient delays in a stochastic model of bargaining. Economic Theory 11, 39-55.

Muthoo, A. (1999). Bargaining theory with applications. Cambridge: Cambridge University Press.

Nash, J. (1953). Two-person cooperative games. Econometrica 21, 128-140.

Osborne, M. and A. Rubinstein (1990). Bargaining and markets. Boston: Academic Press. van Damme, E. (1991). Stability and perfection of Nash equilibria (Second ed.). Berlin: Springer Verlag.

Rubinstein, A. (1982). Perfect equilibrium in a bargaining model. Econometrica 50, 97109.

Shaked, A. and J. Sutton (1984). Involuntary unemployment as a perfect equilibrium in a bargaining model. Econometrica 62, 1351-1364.

Varian, H. (1992). Microeconomic analysis (Third ed.). New York: W. W. Norton \& Company, Inc. 